\title{
TARGET ACHIEVING PORTFOLIO UNDER MODEL MISSPECIFICATION: QUADRATIC OPTIMIZATION FRAMEWORK
}

Abstract. We incorporate model uncertainty into a quadratic portfolio optimization framework. We consider an incomplete continuous time market with a non-tradable stochastic factor. Two stochastic game problems are formulated and solved using Hamilton-Jacobi-Bellman-Isaacs equations. The proof of existence and uniqueness of a solution to the resulting semilinear PDE is also provided. The latter can be used to extend many portfolio optimization results.

1. Introduction. Since Markowitz published his famous paper, quadratic optimization has gained a lot of attraction in asset allocation and active portfolio management. A major weakness of portfolio optimization is its huge sensitivity to estimation errors and model misspecification. Among the articles dedicated to quadratic problems there are also papers which try to incorporate error robustness (or model risk) into the Markowitz framework. However, most of those works concern one-period and static optimization problems. The purpose of this paper is to consider both model uncertainty and quadratic optimization in a dynamic setting with an intertemporal trading. The investor trades between a riskless bond and a risky asset whose price is a diffusion with dynamics affected by a correlated non-tradable stochastic factor. It is worth mentioning that this model includes stochastic volatility models, and it is usually used to describe weather influence on electricity and gas prices. Instead of supposing that the trader knows the exact model, we assume here that he knows that the model belongs to a certain wide

2010 Mathematics Subject Classification: Primary 91G10; Secondary 91A15, 91A23, 49L20, 49N90.

Key words and phrases: robust control, stochastic differential games, model uncertainty, quadratic optimization, quadratic hedging, Knightian uncertainty. 
class of models. Here this class is represented by a set $\mathcal{Q}$ of equivalent measures. Therefore, it is reasonable to consider an optimality criterion based on minimizing the functional

$$
X \mapsto \sup _{Q \in \mathcal{Q}} \mathbb{E}^{Q}(X-D)^{2},
$$

where $D$ is a predefined target to reach. To overcome drawbacks of that model the Maenhout [11 penalty method is introduced. This method is based on the functional

$$
X \mapsto \sup _{Q \in \mathcal{Q}} \mathbb{E}^{Q} h(Q, P)(X-D)^{2},
$$

where $h(Q, P)$ is a multiplicative penalty term which should measure the distance between $Q$ and a reference probability $P$. The measure $P$ can be interpreted as the best guess for the real model and is usually determined by empirical data. The above functionals are motivated by several works concerning robust optimization problems, where a utility function is used rather than the quadratic deviation as an objective function. It is worth mentioning here: Cvitanić and Karatzas [1], Gundel [6], Hernández and Schied [7], Mataramvura and Øksendal [13], Øksendal and Sulem [14], [15], Schied [18], Schied and $\mathrm{Wu}$ [19], Talay and Zheng [20, Zawisza [24]. Part of these papers are based on duality arguments, others use stochastic control theory or differential games and Isaacs equations.

Static and one-period quadratic optimization involving model uncertainty was considered for instance by Tütüncu and Koenig [21] and Goldfarb and Iyengar [5]. In classical dynamic portfolio selection problems (without model risk), the quadratic deviation was used as an objective function by many authors. For recent contributions see for example Xie [22] and the references therein. The classical analogue of our problem was completely solved by Laurent and Pham [10]. To our knowledge, in our paper for the first time model uncertainty is incorporated into a quadratic and continuous time portfolio optimization problem. Our solution to the robust investment problem is closest to those of Hernández and Schied [7] and Zawisza [24]. In the former the authors use a specific class of risk preferences, namely the HARA utility function $\left(U(x)=x^{\gamma}\right)$. This allows them to combine the duality results of Schied and Wu [18] with the stochastic control approach and determine the robust optimal strategy. In the latter paper, an exponential utility $\left(U(x)=-e^{-\gamma x}\right)$ is used as an objective function and the problem of robust hedging is solved by using stochastic differential game theory and Isaacs equations.

In the current paper we also formulate the problem as a stochastic game between the market and the investor. The main result consists of two parts: 
Theorems 3.4 and 4.1 . The first one is a summary of Section 2, where the problem concerning (1.1) is solved. The second one is a solution to (1.2). It is worth pointing out also Proposition 5.1 together with Corollary 5.3, which concern existence and uniqueness of a solution to the resulting PDE and are strong enough to extend many results from the standard optimization portfolio theory (for example Pham [16]).

2. Model description. Let $(\Omega, \mathcal{F}, P)$ be a probability space with filtration $\left(\mathcal{F}_{t}, 0 \leq t \leq T\right)$ (possibly enlarged to satisfy the usual assumptions) generated by two independent Brownian motions $\left(W_{t}^{1}, 0 \leq t \leq T\right),\left(W_{t}^{2}, 0 \leq\right.$ $t \leq T) ; P$ is the best aproximation of a real world probability measure. Our economy consists of two primitive securities: a bond $\left(B_{t}, 0 \leq t \leq T\right)$ and a share $\left(S_{t}, 0 \leq t \leq T\right)$. We also assume that the price of the share is modulated by one non-tradable (but observable) factor $\left(Y_{t}, 0 \leq t \leq T\right)$. This factor can represent an additional source of uncertainty such as: stochastic volatility, varying economic conditions or non-financial risk (for instance weather risk). The processes mentioned above are solutions of the system of stochastic differential equations

$$
\left\{\begin{array}{l}
d B_{t}=r B_{t} d t \\
d S_{t}=b\left(Y_{t}\right) S_{t} d t+\sigma\left(Y_{t}\right) S_{t} d W_{t}^{1}, \\
d Y_{t}=g\left(Y_{t}\right) d t+a\left(Y_{t}\right)\left(\rho d W_{t}^{1}+\bar{\rho} d W_{t}^{2}\right), \quad \rho^{2}+\bar{\rho}^{2}=1 .
\end{array}\right.
$$

The coefficients $b, \sigma>0, g, a$ are continuous functions and they are assumed to satisfy all the necessary regularity conditions, in order to guarantee that there exists a unique strong solution to 2.1). The coefficient $r>0$ is a deterministic interest rate and $\rho \in[-1,1]$ is a correlation coefficient. The assumption of time-independent coefficients is for notational convenience only and can easily be relaxed.

We assume that the investor faces a model risk. This means that the model (probability measure) is not precisely known and the investor knows only a class of possible measures. Following Cvitanić and Karatzas [1 and Hernández and Schied [7], we will consider the class

$$
\mathcal{Q}:=\left\{Q \sim P \mid \frac{d Q}{d P}=\mathcal{E}\left(\int \eta_{t 1} d W_{t}^{1}+\eta_{t 2} d W_{t}^{2}\right)_{T},\left(\eta_{1}, \eta_{2}\right) \in \mathcal{M}\right\},
$$

where $\mathcal{E}(\cdot)_{t}$ denotes the Doléans-Dade exponential and $\mathcal{M}$ denotes the set of all bounded, progressively measurable processes $\eta=\left(\eta_{1}, \eta_{2}\right)$ taking values in a fixed set $\Gamma \subset \mathbb{R}^{2}$. Usually it is assumed that $\Gamma$ is a compact and convex set. However in Section 3 we allow $\Gamma$ to be $\mathbb{R}^{2}$. The measure determined by $\eta \in \mathcal{M}$ is denoted later by $Q^{\eta}$, and the corresponding expectation by $\mathbb{E}^{\eta}$. 
The dynamics of the investor's wealth process $\left(\bar{X}_{t}^{\bar{\pi}}, 0 \leq t \leq T\right)$ is given by the stochastic differential equation

$$
\left\{\begin{array}{l}
d \bar{X}_{t}=\left(r \bar{X}_{t}+\bar{\pi}_{t}\left(b\left(Y_{t}\right)-r\right)\right) d t+\bar{\pi}_{t} \sigma\left(Y_{t}\right) d W_{t}^{1}, \\
\bar{X}_{s}=\bar{x}
\end{array}\right.
$$

where $\bar{x}$ denotes the current wealth of the investor.

Definition 2.1. A control (or strategy) $\bar{\pi}=\left(\bar{\pi}_{s}, t \leq s \leq T\right)$ is admissible on the time interval $[t, T]$, written $\bar{\pi} \in \mathcal{A}_{t}$, if it satisfies the following assumptions:

(1) $\bar{\pi}$ is progressively measurable with respect to the filtration $\left(\mathcal{F}_{s}, t \leq\right.$ $s \leq T)$

(2) the stochastic process 2.2 exists and

$$
\mathbb{E}_{y, t}^{\eta} \sup _{t \leq s \leq T}\left(\bar{X}_{s}^{\bar{\pi}}\right)^{2}<\infty \quad \text { for all } y \in \mathbb{R} \text { and } \eta \in \mathcal{M} .
$$

The admissible control $\bar{\pi}$ can be interpreted as part of the wealth invested in $S_{t}$. More precisely if $h_{t}$ denotes the number of shares in the portfolio, then $\bar{\pi}_{t}=h_{t} S_{t}$. Note that $\bar{\pi}$ as well as the portfolio wealth $\bar{X}_{T}^{\bar{\pi}}$ are allowed to be negative.

Formulation of the problem. Let us define an objective function

$$
\mathcal{J}^{\bar{\pi}, \eta}(\bar{x}, y, t):=\mathbb{E}_{\bar{x}, y, t}^{\eta}\left(\bar{X}_{T}^{\bar{\pi}}-D\right)^{2} .
$$

The investor's objective is to

$$
\operatorname{minimize} \sup _{\eta \in \mathcal{M}} \mathcal{J}^{\bar{\pi}, \eta}(\bar{x}, y, t)
$$

over the class $\mathcal{A}_{t}$ of admissible strategies. This means that the aim of the investor is to control the investment of a given wealth via dynamic asset allocation, taking into account model uncertainty, in such a way that the performance of the investment follows as closely as possible the prescribed target $D$. The quadratic function does not allow the investor's wealth to outperform the target, and this can be considered as a drawback to the model. However, once the target is reached, there is no reason for further exposure to risk, and therefore it is no good to have any additional surplus. The idea that people act by following subjective targets is accepted in the decision theory literature (see for example the prospect theory of Kahneman and Tversky [9]).

It is convenient for us to introduce $T$-forward values of $\bar{\pi}$ and $\bar{X}_{t}^{\bar{\pi}}$. Let

$$
X_{t}^{\pi}:=e^{r(T-t)} \bar{X}_{t}^{\bar{\pi}}, \quad \pi_{t}:=e^{r(T-t)} \bar{\pi}_{t} .
$$

Instead of (2.3) we use the objective

$$
J^{\pi, \eta}(x, y, t):=\mathbb{E}_{x, y, t}^{\eta}\left(X_{T}^{\pi}-D\right)^{2},
$$


where the dynamics of the new wealth process $X^{\pi}$ is given by

$$
d X_{t}=\pi_{t}\left(b\left(Y_{t}\right)-r\right) d t+\pi_{t} \sigma\left(Y_{t}\right) d W_{t}^{1} .
$$

Note that $\mathcal{J}^{\bar{\pi}, \eta}(x, y, t)=J^{\bar{\pi}, \eta}(\bar{x}, y, t)$ and $\pi$ is admissible if and only if $\bar{\pi}$ is admissible.

REMARK. Using (2.3), we are able to cover also the objective

$$
\mathbb{E}_{x, y, t}^{\eta} X_{T}^{\pi}-\beta \mathbb{E}_{x, y, t}^{\eta}\left(X_{T}^{\pi}-B\right)^{2}=-\beta \mathbb{E}_{x, y, t}^{\eta}\left(X_{T}^{\pi}-\left(B+\frac{1}{2 \beta}\right)\right)^{2}+B+\frac{1}{4 \beta} .
$$

By using such an objective, we assume that the investor wants to maximize his expected wealth while controlling the risk which is measured by a quadratic deviation from a predefined target $B$. This is a modification of the Markowitz problem, where the risk is usually measured by the variance. Such a modification is accepted in financial and insurance optimization theory (see Delong [2] and references therein); moreover it is usually used as an auxiliary problem in finding a solution to the original Markowitz problem (see Zhou and Li 25]). It should also be mentioned that this modification leads to time-consistent optimal strategies, which is not generally the case for solutions to the continuous time Markowitz problem.

The problem (2.4) is considered as a zero-sum stochastic differential game problem. The measure $Q$ is the control of player 1 (the "market"), while the portfolio $\pi$ is the control of player 2 (the "investor"). We are looking for a saddle point $\left(\pi^{*}, \eta^{*}\right) \in \mathcal{A}_{t} \times \mathcal{M}$ and a value function $V(x, y, t)$ such that

$$
J^{\pi^{*}, \eta}(x, y, t) \leq J^{\pi^{*}, \eta^{*}}(x, y, t) \leq J^{\pi, \eta^{*}}(x, y, t),
$$

and

$$
V(x, y, t)=J^{\pi^{*}, \eta^{*}}(x, y, t) .
$$

Stochastic control theory gives us a motivation to seek optimal strategies in the feedback form $\left(\left(\pi\left(X_{t}, Y_{t}, t\right), \eta\left(X_{t}, Y_{t}, t\right)\right), 0 \leq t \leq T\right)$ ( $\eta$ determines the measure $\left.Q^{\eta}\right), \pi(x, y, t), \eta(x, y, t)$ are Borel functions and $X_{t}$ and $Y_{t}$ are solutions to the system

$$
\left\{\begin{array}{l}
d X_{t}=\pi\left(X_{t}, Y_{t}, t\right)\left(b\left(Y_{t}\right)-r\right) d t+\pi\left(X_{t}, Y_{t}, t\right) \sigma\left(Y_{t}\right) d W_{t}^{1}, \\
d Y_{t}=g\left(Y_{t}\right) d t+a\left(Y_{t}\right)\left(\rho d W_{t}^{1}+\bar{\rho} d W_{t}^{2}\right) .
\end{array}\right.
$$

Such controls are often called Markov controls and are denoted simply by $(\pi(x, y, t), \eta(x, y, t))$.

3. HJBI equations, Verification Theorem and solution to the minimax problem. The robust investment problem stated in the previous section can be solved by applying the HJB theory. In this section we establish a link between Hamilton-Jacobi-Bellman-Isaacs equations and a saddle point of our initial problem. 
Let $\mathcal{L}^{\pi, \eta}$ denote the differential operator given by

$$
\begin{aligned}
\mathcal{L}^{\pi, \eta} V(x, y, t):= & V_{t}+\frac{1}{2} a^{2}(y) V_{y y}+\frac{1}{2} \pi^{2} \sigma^{2}(y) V_{x x}+\rho \pi \sigma(y) a(y) V_{x y} \\
& +\pi\left(b(y)-r+\eta_{1} \sigma(y)\right) V_{x}+\left(\rho \eta_{1}+\bar{\rho} \eta_{2}\right) a(y) V_{y}+g(y) V_{y} .
\end{aligned}
$$

We will use a suitable version of the Verification Theorem. Its proof in a more general form is presented in the Appendix.

TheOREM 3.1. Suppose there exists a function $V \in \mathcal{C}^{2,2,1}\left(\mathbb{R}^{2} \times[0, T)\right) \cap$ $\mathcal{C}\left(\mathbb{R}^{2} \times[0, T]\right)$ and an admissible Markov control $\left(\pi^{*}(x, y, t), \eta^{*}(x, y, t)\right)$ such that

$$
\begin{aligned}
& \mathcal{L}^{\pi^{*}(x, y, t), \eta} V(x, y, t) \leq 0, \\
& \mathcal{L}^{\pi, \eta^{*}(x, y, t)} V(x, y, t) \geq 0, \\
& \mathcal{L}^{\pi^{*}(x, y, t), \eta^{*}(x, y, t)} V(x, y, t)=0, \\
& V(x, y, T)=(x-D)^{2} \quad \text { for all } \eta \in \Gamma, \pi \in \mathbb{R},(x, y, t) \in \mathbb{R}^{2} \times[0, T),
\end{aligned}
$$

and

$$
\begin{aligned}
\mathbb{E}_{x, y, t}^{\eta}\left(\sup _{t \leq s \leq T}\left|V\left(X_{s}^{\pi}, Y_{s}, s\right)\right|\right)<\infty & \\
& \text { for all }(x, y, t) \in \mathbb{R}^{2} \times[0, T], \pi \in \mathcal{A}_{t}, \eta \in \mathcal{M} .
\end{aligned}
$$

Then

$$
J^{\pi^{*}, \eta}(x, y, t) \leq V(x, y, t) \leq J^{\pi, \eta^{*}}(x, y, t) \quad \text { for all } \pi \in \mathcal{A}_{t}, \eta \in \mathcal{M},
$$

and

$$
V(x, y, t)=J^{\pi^{*}, \eta^{*}}(x, y, t) .
$$

Let us point out that conditions (3.1)-3.4 hold if the upper and the lower Hamilton-Jacobi-Bellman-Isaacs equations are satisfied:

$$
\begin{aligned}
\min _{\pi \in \mathbb{R}} \max _{\eta \in \Gamma} \mathcal{L}^{\pi, \eta} V(x, y, t) & =\max _{\eta \in \Gamma} \min _{\pi \in \mathbb{R}} \mathcal{L}^{\pi, \eta} V(x, y, t)=0, \\
V(x, y, T) & =(x-D)^{2} .
\end{aligned}
$$

To find the saddle point it is more convenient for us to use the lower Isaacs equation

$$
\max _{\eta \in \Gamma} \min _{\pi \in \mathbb{R}} \mathcal{L}^{\pi, \eta} V(x, y, t)=0 .
$$

Once we verify that it has a unique solution $V$, it is also necessary to prove that $V$ is also a solution to the upper equation. To do that we use a minimax theorem proved by Fan ([3, Theorem 2]).

TheOREM 3.2. Let $X$ be a compact Hausdorff space and $Y$ an arbitrary set (not topologized). Let $f$ be a real-valued function on $X \times Y$ such that, for every $\eta \in Y, f(\pi, \eta)$ is lower semicontinuous on $X$. If $f$ is concave on $X$ 
and convex on $Y$, then

$$
\max _{\eta \in X} \inf _{\pi \in Y} f(\pi, \eta)=\inf _{\pi \in Y} \max _{\eta \in X} f(\pi, \eta) .
$$

Derivation of the optimal strategy. As announced, to find explicit forms of the saddle point $\left(\eta^{*}(x, y, t), \pi^{*}(x, y, t)\right)$, we start with the lower Isaacs equation

$$
\max _{\eta \in \Gamma} \min _{\pi \in \mathbb{R}} \mathcal{L}^{\pi, \eta} V(x, y, t)=0
$$

i.e.

$$
\begin{aligned}
V_{t}+\frac{1}{2} a^{2}(y) V_{y y}+\max _{\left(\eta_{1}, \eta_{2}\right) \in \Gamma} \min _{\pi \in \mathbb{R}}\left(\frac{1}{2} \pi^{2} \sigma^{2}(y) V_{x x}+\rho \pi \sigma(y) a(y) V_{x y}\right. \\
\left.\quad+\pi\left(b(y)-r+\eta_{1} \sigma\right) V_{x}+\left(\rho \eta_{1}+\bar{\rho} \eta_{2}\right) a(y) V_{y}\right)+g(y) V_{y}=0
\end{aligned}
$$

with the terminal condition $V(x, y, T)=(x-D)^{2}$.

Note that if there exists $V \in \mathcal{C}^{2,2,1}\left(\mathbb{R}^{2} \times[0, T)\right)$ with $V_{x x}>0$, then the minimum over $\pi$ in $(3.6)$ is well defined and achieved at

$$
\pi^{*}(x, y, t, \eta)=-\frac{\rho a(y)}{\sigma(y)} \frac{V_{x y}}{V_{x x}}-\frac{b(y)+\eta_{1} \sigma(y)}{\sigma^{2}(y)} \frac{V_{x}}{V_{x x}} .
$$

The appearance of the terminal condition $V(x, y, T)=(x-D)^{2}$ motivates us to seek a solution of the form

$$
V(x, y, t):=(x-D)^{2} F(y, t) .
$$

Substituting (3.7) and 3.8 in 3.6 yields

$$
\pi^{*}(x, y, t, \eta)=-\frac{\rho a(y)(x-D)}{\sigma(y)} \frac{F_{y}}{F}-\frac{\left(\lambda(y)+\eta_{1}\right)(x-D)}{\sigma(y)},
$$

where $\lambda(y):=\frac{b(y)-r}{\sigma(y)}$ and $F$ should solve

$$
\begin{aligned}
F_{t}+\frac{1}{2} a^{2}(y) F_{y y}-\rho^{2} a^{2}(y) \frac{F_{y}^{2}}{F}+(g(y)-2 \rho a(y) \lambda(y)) F_{y} \\
\quad+\max _{\eta \in \Gamma}\left(-\eta_{1} \rho a(y) F_{y}+\eta_{2} \bar{\rho} a(y) F_{y}-\left(\eta_{1}+\lambda(y)\right)^{2} F\right)=0
\end{aligned}
$$

together with the terminal condition $F(y, T)=1$.

Existence and uniqueness of a solution to equation $(3.10)$ is studied in Section 4.

If there exists a smooth solution to 3.10 then in order to determine the saddle point $\left(\pi^{*}(x, y, t), \eta^{*}(x, y, t)\right)$ we should find a Borel measurable $\eta^{*}(x, y, t)$ such that

$$
\max _{\eta \in \Gamma} \min _{\pi \in \mathbb{R}} \mathcal{L}^{\pi, \eta} V(x, y, t)=\min _{\pi \in \mathbb{R}} \mathcal{L}^{\pi, \eta^{*}(x, y, t)} V(x, y, t)
$$


and a Borel measurable $\pi^{*}(x, y, t)$ such that

$$
\min _{\pi \in \mathbb{R}} \max _{\eta \in \Gamma} \mathcal{L}^{\pi, \eta} V(x, y, t)=\max _{\eta \in \Gamma} \mathcal{L}^{\pi^{*}(x, y, t), \eta} V(x, y, t) .
$$

From 3.6-3.10, it follows that $\eta^{*}(x, y, t)$ does not depend on $x$ and is equal to a maximizer of 3.10. Moreover, $\pi^{*}(x, y, t)=\pi^{*}\left(x, y, t, \eta^{*}(y, t)\right)$, where $\pi^{*}(x, y, t, \eta)$ is given by (3.9). The last claim is a consequence of the minimax equality

$\max _{\eta \in \Gamma} \min _{\pi \in \mathbb{R}} \mathcal{L}^{\pi, \eta} V(x, y, t)=\min _{\pi \in \mathbb{R}} \max _{\eta \in \Gamma} \mathcal{L}^{\pi, \eta} V(x, y, t)=\mathcal{L}^{\pi^{*}(x, y, t), \eta^{*}(x, y, t)} V(x, y, t)$, and the fact that $\mathcal{L}^{\pi^{*}(x, y, t), \eta^{*}(x, y, t)} V(x, y, t)=\min _{\pi} \mathcal{L}^{\pi, \eta^{*}(x, y, t)} V(x, y, t)$ and therefore $\pi^{*}(x, y, t)$ is a unique solution to the equation

$$
\mathcal{L}^{\pi, \eta^{*}(x, y, t)} V(x, y, t)=\mathcal{L}^{\pi^{*}(x, y, t), \eta^{*}(x, y, t)} V(x, y, t) .
$$

We can now summarize our preparatory calculations.

Corollary 3.3. Suppose that there exists a strictly positive solution $F$ to problem (3.10) and let the maximum in 3.10 be attained at $\eta^{*}(y, t)=$ $\left(\eta_{1}^{*}(y, t), \eta_{2}^{*}(y, t)\right)$. Then

$$
\mathcal{L}^{\pi^{*}, \eta} V(x, y, t) \leq \mathcal{L}^{\pi^{*}, \eta^{*}} V(x, y, t)=0 \leq \mathcal{L}^{\pi, \eta^{*}} V(x, y, t)
$$

where

$$
\begin{aligned}
V(x, y, t) & =(x-D)^{2} F(y, t) \\
\pi^{*}(x, y, t) & =-\frac{\rho a(y)(x-D)}{\sigma(y)} \frac{F_{y}}{F}-\frac{\left(\lambda(y)+\eta_{1}^{*}(y, t)\right)(x-D)}{\sigma(y)} .
\end{aligned}
$$

If $a \equiv 1, g$ is Lipschitz continuous, and $\lambda$ is Lipschitz continuous and bounded, then by Proposition 5.3, all assertions of Corollary 3.3 are satisfied.

THEOREM 3.4. Suppose $a, a_{y}, g, \lambda$ are Lipschitz continuous functions, $a, \lambda$ are bounded and $a(y)>\varepsilon>0$. Then there exists a saddle point $\left(\pi^{*}(x, y, t), \eta^{*}(x, y, t)\right)$ such that

$$
\pi^{*}(x, y, t)=-\frac{\rho a(y)(x-D)}{\sigma(y)} \frac{F_{y}}{F}-\frac{\left(\lambda(y)+\eta_{1}^{*}(y, t)\right)(x-D)}{\sigma(y)},
$$

where $\eta^{*}$ is a Borel measurable function which realizes the maximum in 3.10 .

Proof. First we need to show that we can limit ourselves to the case $a \equiv 1$. Notice that, if $a$ is $C^{1}$ and bounded, and $a(y)>\varepsilon>0$, then applying the Itô formula to the function $f(y):=\int_{0}^{y} \frac{1}{a(z)} d z$ and to the process $Y$ with the dynamics

$$
d Y_{t}=g\left(Y_{t}\right) d t+a\left(Y_{t}\right)\left(\rho d W_{t}^{1}+\bar{\rho} d W_{t}^{2}\right)
$$


we can rewrite the dynamics of our model 2.1 as follows:

$$
\left\{\begin{array}{l}
d B_{t}=r B_{t} d t \\
d S_{t}=b\left(Z_{t}\right) S_{t} d t+\sigma\left(Z_{t}\right) S_{t} d W_{t}^{1}, \\
d Z_{t}=\left(\frac{g\left(Y_{t}\right)}{a\left(Y_{t}\right)}-\frac{1}{2} a_{y}\left(Y_{t}\right)\right) d t+\left(\rho d W_{t}^{1}+\bar{\rho} d W_{t}^{2}\right), \quad Y_{t}=f^{-1}\left(Z_{t}\right) .
\end{array}\right.
$$

Moreover if $\lambda, g / a, a_{y}$ are bounded or Lipschitz continuous then the same is true for $\lambda\left(f^{-1}(z)\right), g\left(f^{-1}(z)\right) / a\left(f^{-1}(z)\right), a_{y}\left(f^{-1}(z)\right)$.

The second part of the proof needs the assumption $a \equiv 1$. It follows from Proposition 5.3 that there exists a positive function $F$, bounded away from zero and bounded together with the first $y$-derivative, which satisfies

$$
\begin{aligned}
F_{t}+\frac{1}{2} a^{2}(y) F_{y y}-\rho^{2} a^{2}(y) \frac{F_{y}^{2}}{F}+(g(y)-2 \rho a(y) \lambda(y)) F_{y} \\
\quad+\max _{\eta \in \Gamma}\left(-\eta_{1} \rho a(y) F_{y}+\eta_{2} \bar{\rho} a(y) F_{y}-\left(\eta_{1}+\lambda(y)\right)^{2} F\right)=0
\end{aligned}
$$

together with the terminal condition $F(y, T)=1$.

By the classical measurable selection theorem there exists a Borel measurable $\eta^{*}(y, t) \in \Gamma$ that achieves the maximum in (3.11).

If we set

$$
\begin{aligned}
V(x, y, t) & :=(x-D)^{2} F(y, t) \\
\pi^{*}(x, y, t) & :=-\frac{\rho a(y)(x-D)}{\sigma(y)} \frac{F_{y}}{F}-\frac{\left(\lambda(y)+\eta_{1}^{*}(y, t)\right)(x-D)}{\sigma(y)},
\end{aligned}
$$

then due to Corollary 3.3, we only need to prove that $\left(\pi^{*}(x, y, t), \eta^{*}(x, y, t)\right)$ is an admissible Markov saddle point and condition (3.5) holds. Let

$$
\zeta(y, t):=-\frac{\rho a(y)}{\sigma(y)} \frac{F_{y}}{F}-\frac{\lambda(y)+\eta_{1}^{*}(y, t)}{\sigma(y)} .
$$

Note that $\zeta \cdot(b-r)$ and $\zeta \cdot \sigma$ are bounded functions since $\lambda$ and $\lambda^{2}$ are bounded. Therefore, the process $Z_{t}:=X_{t}^{\pi^{*}}-D$ is a unique solution to the equation

$$
d Z_{t}=\zeta\left(Y_{t}, t\right)\left(b\left(Y_{t}\right)-r\right) Z_{t} d t+\eta_{1} \zeta\left(Y_{t}, t\right) \sigma\left(Y_{t}\right) Z_{t} d t+\zeta\left(Y_{t}, t\right) \sigma\left(Y_{t}\right) Z_{t} d W^{\eta},
$$

where $W^{\eta}$ denotes the Brownian motion determined by the Girsanov transformation and the measure $Q^{\eta}$. This is a linear equation with bounded stochastic coefficients, which implies that

$$
\mathbb{E}_{x, y, t}^{\eta}\left(\sup _{t \leq s \leq T}\left(X_{s}^{\pi^{*}}-D\right)^{2}\right)<\infty
$$

for all $\eta \in \mathcal{M}$. This confirms the admissibility of $\pi^{*}(x, y, t)$. 
Condition 3.5 is satisfied because $F$ is bounded and for any admissible strategy $\pi$ and for any $x, y \in \mathbb{R}, t \geq 0$,

$$
\mathbb{E}_{x, y, t}^{\eta}\left(\sup _{t \leq s \leq T}\left|V\left(X_{s}^{\pi}, Y_{s}, s\right)\right|\right)=\mathbb{E}_{x, y, t}^{\eta}\left(\sup _{t \leq s \leq T}\left(X_{s}^{\pi}-D\right)^{2}\left|F\left(Y_{s}, s\right)\right|\right)<\infty .
$$

Two prominent examples. We can apply our main result to the following stochastic volatility models:

- Scott model:

$$
\left\{\begin{array}{l}
d S_{t}=b d t+\sqrt{e^{\gamma Y_{t}}+\varepsilon} d W_{t}^{1}, \quad \varepsilon>0, \\
d Y_{t}=\left(a-\theta Y_{t}\right) d t+\rho d W_{t}^{1}+\bar{\rho} d W_{t}^{2} .
\end{array}\right.
$$

- Stein and Stein model:

$$
\left\{\begin{array}{l}
d S_{t}=b d t+\left(\left|Y_{t}\right|+\varepsilon\right) d W_{t}^{1}, \quad \varepsilon>0 \\
d Y_{t}=\left(a-\theta Y_{t}\right) d t+\rho d W_{t}^{1}+\bar{\rho} d W_{t}^{2}
\end{array}\right.
$$

Note that in fact we propose an $\varepsilon$-modification of the original models. We need it to ensure Lipschitz continuity and boundedness of the market price of risk $\lambda$.

4. Penalty method. According to the worst-case method presented in the previous sections, all scenarios (all measures $Q$ ) have equal chance to occur in reality. This might result in putting too much trust in very pessimistic scenarios and consequently in very pessimistic solutions. For example, suppose that the market price of risk satisfies $-\lambda(y) \in \operatorname{Pr}_{1} \Gamma$ for all $y \in \mathbb{R}$ $\left(\operatorname{Pr}_{1}\right.$ is the projection on the first coordinate). In this case it is easy to check that $F \equiv 1$ is a solution to (3.11). Therefore $\eta_{1}^{*}(y, t)=-\lambda(y)$ and $\pi^{*}(x, y, t)=0$ is a saddle point of the game.

Therefore it might be reasonable to modify our method so as to take into account the fact that measures which are more distant from the reference measure $P$ are less likely to occur in reality. To do that, the minimax criterion is rewritten using a penalty function $H(Q, P)$. The task of $H(Q, P)$ is to measure the distance between two measures and usually it is assumed that the penalty is equal to the entropic function $\ln \frac{d Q}{d P}$. In the quadratic case it can be useful to use an objective of the form

$$
\mathbb{E}_{x, y}^{\eta}\left(\left(X_{T}^{\pi}-D\right)^{2}-\theta H\left(Q^{\eta}, P\right)\right)=\mathbb{E}_{x, y}^{\eta}\left(\left(X_{T}^{\pi}-D\right)^{2}-\theta \ln \frac{d Q^{\eta}}{d P}\right) .
$$

Note that in our model

$$
\mathbb{E}^{\eta} \ln \frac{d Q^{\eta}}{d P}=\mathbb{E}^{\eta} \frac{1}{2} \int_{0}^{T}\left|\eta_{s}\right|^{2} d s .
$$

The parameter $\theta>0$ can be interpreted as an ambiguity aversion (model risk aversion) index. The lower $\theta>0$, the higher the degree of ambiguity 
aversion. The objective function (4.1) leads to the following Isaacs equation:

$$
\begin{aligned}
& \max _{\eta \in \Gamma} \min _{\pi \in \mathbb{R}}\left(V_{t}+\frac{1}{2} a^{2}(y) V_{y y}+\frac{1}{2} \pi^{2} \sigma^{2}(y) V_{x x}+\rho \pi \sigma(y) a(y) V_{x y}\right. \\
& \left.+\pi\left(b(y)-r+\eta_{1} \sigma(y)\right) V_{x}+\left(\rho \eta_{1}+\bar{\rho} \eta_{2}\right) a(y) V_{y}+g(y) V_{y}-\frac{1}{2} \theta\left(\eta_{1}^{2}+\eta_{2}^{2}\right)\right)=0 .
\end{aligned}
$$

Unfortunately, it is impossible to find a tractable analytical solution to this equation. To overcome this obstacle we adopt here Maenhout's [11] idea, i.e. we replace the penalty term $\theta\left(\eta_{1}^{2}+\eta_{2}^{2}\right)$ by $\theta\left(\eta_{1}^{2}+\eta_{2}^{2}\right) V$. This method is accepted in financial literature and it was applied for example to price catstrophy bonds by Zhu [26] or to value defaultable bonds by Jaimungal and Sigloch [8]. We can also define a new objective function which corresponds to the resulting equation:

$$
\mathcal{K}^{\pi, \eta}(x, y, t):=\mathbb{E}_{x, y, t}^{\eta}\left(e^{-\frac{1}{2} \theta \int_{t}^{T}\left|\eta_{s}\right|^{2} d s}\left(X_{T}^{\pi}-D\right)^{2}\right) .
$$

The term $e^{-\frac{1}{2} \theta \int_{t}^{T}\left|\eta_{s}\right|^{2} d s}$ may be treated as a multiplicative form of a penalty function. Our task is to find a saddle point for 4.2 . The solution to this game can be found just as the solution to (2.4). The Isaacs equation for 4.2 has the form

$$
\begin{aligned}
& \max _{\eta \in \Gamma} \min _{\pi \in \mathbb{R}}\left(V_{t}+\frac{1}{2} a^{2}(y) V_{y y}+\frac{1}{2} \pi^{2} \sigma^{2}(y) V_{x x}+\rho \pi \sigma(y) a(y) V_{x y}\right. \\
& \left.+\pi\left(b(y)-r+\eta_{1} \sigma(y)\right) V_{x}+\left(\rho \eta_{1}+\bar{\rho} \eta_{2}\right) a(y) V_{y}+g(y) V_{y}-\frac{1}{2} \theta\left(\eta_{1}^{2}+\eta_{2}^{2}\right) V\right)=0 .
\end{aligned}
$$

REMARK. Note that for this equation one can also allow the set $\Gamma$ to be $\mathbb{R}^{2}$. In that case the magnitude of ambiguity aversion is only controlled by $\theta>0$. In our opinion this is a convenient assumption and it is discussed later with a few more details.

The Verification Theorem for $(4.2)$ is given in the Appendix. To solve the above equation the well known substitution $V(x, y, t)=(x-D)^{2} F(y, t)$ can be applied to obtain a candidate for optimal investment policy:

$$
\pi^{*}(x, y, t)=-\frac{\rho a(y)(x-D)}{\sigma(y)} \frac{F_{y}}{F}-\frac{\left(\lambda(y)+\eta_{1}^{*}(y, t)\right)(x-D)}{\sigma(y)},
$$

where $F$ is the solution to the equation

$$
\begin{gathered}
F_{t}+\frac{1}{2} a^{2}(y) F_{y y}-\rho^{2} a^{2}(y) \frac{F_{y}^{2}}{F}+(g(y)-2 \rho a(y) \lambda(y)) F_{y} \\
+\max _{\eta \in \Gamma}\left(-\eta_{1} \rho a(y) F_{y}+\bar{\rho} a(y) \eta_{2} F_{y}-\left(\eta_{1}+\lambda(y)\right)^{2} F-\frac{1}{2} \theta\left(\eta_{1}^{2}+\eta_{2}^{2}\right) F\right)=0
\end{gathered}
$$

with the terminal condition $F(y, T)=1$. 
If we assume that $\Gamma=\mathbb{R}^{2}$ then the solution to the problem is even more tractable because it is given by

$$
\begin{aligned}
\pi^{*}(x, y, t) & =-\frac{(1+\theta) \rho a(y)(x-D)}{(2+\theta) \sigma(y)} \frac{F_{y}}{F}-\frac{\theta \lambda(y)(x-D)}{(2+\theta) \sigma(y)}, \\
\eta_{1}^{*}(x, y, t) & =-\frac{2 \lambda(y)}{2+\theta}-\frac{\rho a(y)}{2+\theta} \frac{F_{y}}{F}, \\
\eta_{2}^{*}(x, y, t) & =\frac{a(y) \bar{p}}{\theta} \frac{F_{y}}{F},
\end{aligned}
$$

where $F$ is the unique classical solution to

$$
\begin{aligned}
F_{t}+\frac{1}{2} a^{2}(y) F_{y y}+\left[\frac{\rho^{2}}{2(2+\theta)}+\frac{\bar{\rho}^{2}}{2 \theta}-\rho^{2}\right] a^{2}(y) \frac{F_{y}^{2}}{F} \\
\\
+(g(y)-(1+\theta) \rho a(y) \lambda(y)) F_{y}-\frac{1}{2} \theta \lambda^{2}(y) F=0
\end{aligned}
$$

with the terminal condition $F(y, T)=1$.

In the following theorem we summarize the findings of this section. The proof is omitted since it is the repetition of the steps of the proof of Theorem 3.4 .

TheOREM 4.1. Suppose that $a, a_{y}, g, \lambda$ are Lipschitz continuous functions, $a, \lambda$ are bounded and $a(y)>\varepsilon>0$. Then there exists a Markov saddle point $\left(\pi^{*}(x, y, t), \eta^{*}(y, t)\right)$ for the objective 4.2 such that

$$
\pi^{*}(x, y, t)=-\frac{\rho a(y)(x-D)}{\sigma(y)} \frac{F_{y}}{F}-\frac{\left(\lambda(y)+\eta_{1}^{*}(y, t)\right)(x-D)}{\sigma(y)},
$$

where $F$ is the unique classical solution to 4.3) (or 4.4) if $\Gamma=\mathbb{R}^{2}$ ), bounded together with $F_{y}$, and the maximum in 4.3 is achieved at $\eta^{*}(y, t)$.

5. Smooth solution to the resulting PDE. In this section, we use stochastic methods to derive existence and uniqueness results for classical solutions of semilinear parabolic partial differential equations which play a key role in solving our initial problem. To the best of our knowledge, the subsequent results on classical solutions have not appeared in print so far under the assumptions given here. Moreover, they are strong enough to extend results of Hernández and Schied [7] and Schied [18] and even Pham [16], because to ensure existence and uniqueness of a solution, we do not need the differentiability of the equation coefficients. Let us recall the shape of our equation once more:

$$
\begin{aligned}
& F_{t}+\frac{1}{2} a^{2}(y) F_{y y}-\rho^{2} a^{2}(y) \frac{F_{y}^{2}}{F}+(g(y)-2 \rho a(y) \lambda(y)) F_{y} \\
+ & \max _{\eta \in \Gamma}\left(-\eta_{1} \rho a(y) F_{y}+\bar{\rho} a(y) \eta_{2} F_{y}-\left(\eta_{1}+\lambda(y)\right)^{2} F-\theta\left(\eta_{1}^{2}+\eta_{2}^{2}\right) F\right)=0
\end{aligned}
$$

with the terminal condition $F(y, T)=1$. 
First we apply the exponential transformation $F(y, t)=e^{\alpha(y, t)}$. Straightforward calculations show that $\alpha$ should solve

$$
\begin{aligned}
& \alpha_{t}+\frac{1}{2} a^{2}(y) \alpha_{y y}+\left(1 / 2-\rho^{2}\right) a^{2}(y) \alpha_{y}^{2}+(g(y)-2 \rho a(y) \lambda(y)) \alpha_{y} \\
& \quad+\max _{\eta \in \Gamma}\left(-\eta_{1} \rho a(y) \alpha_{y}+\bar{\rho} a(y) \eta_{2} \alpha_{y}-\left(\eta_{1}+\lambda(y)\right)^{2}-\theta\left(\eta_{1}^{2}+\eta_{2}^{2}\right)\right)=0
\end{aligned}
$$

together with the terminal condition $\alpha(y, T)=0$.

REMARK. The nonlinear term $F_{y}^{2} / F$ can also be removed from 5.1) by applying transformations of the form $F(y, t)=(\alpha(y, t))^{\delta}$ (see Zariphopoulou [23], Zawisza [24]). However this substitution works only in the case when $y$ is one-dimensional. As we would like to have also a possibility to extend our problem to some multifactor models, we apply a more sophisticated reasoning.

Let us rewrite equation 5.2 in a more general form:

$$
\alpha_{t}+\frac{1}{2} a^{2}(y) \alpha_{y y}+c a^{2}(y) \alpha_{y}^{2}+f(y) \alpha_{y}+\max _{\eta \in \Gamma}\left(i(y, \eta) \alpha_{y}+h(y, \eta)\right)=0 .
$$

Assume that there exists a solution $\alpha$ to 5.3 with bounded derivative $\alpha_{y}$. In this case there exists $R>0$ such that

$$
\begin{array}{ll}
\max _{q \in[-R, R]}\left(-c a^{2}(y) q^{2}+2 c a^{2}(y) \alpha_{y} q\right)=c a^{2}(y) \alpha_{y}^{2} & \text { for } c \geq 0, \\
\min _{q \in[-R, R]}\left(-c a^{2}(y) q^{2}+2 c a^{2}(y) \alpha_{y} q\right)=c a^{2}(y) \alpha_{y}^{2} & \text { for } c<0 .
\end{array}
$$

Therefore, it is worth considering equations of the form

$$
\begin{aligned}
\alpha_{t}+\frac{1}{2} a^{2}(y) \alpha_{y y}+\min _{q \in[-R, R]}\left(-c a^{2}(y) q^{2}+2 c a^{2}(y) \alpha_{y} q\right)+f(y) \alpha_{y} & \\
& +\max _{\eta \in \Gamma}\left(i(y, \eta) \alpha_{y}+h(y, \eta)\right)=0 .
\end{aligned}
$$

Up to the end of this section we consider only the case $c<0$. The reasoning for $c \geq 0$ is analogous.

Proposition 5.1. Suppose that $a, f, h, i$ are continuous functions and there exists $L \geq 1$ such that

$$
\begin{aligned}
\left|f\left(y_{1}\right)-f\left(y_{2}\right)\right| & \leq L\left|y_{1}-y_{2}\right|, \\
\left|i\left(y_{1}, \eta\right)-i\left(y_{2}, \eta\right)\right| & \leq L\left|y_{1}-y_{2}\right|, \\
\left|h\left(y_{1}, \eta\right)-h\left(y_{2}, \eta\right)\right| & \leq L\left|y_{1}-y_{2}\right|, \\
\left|a^{2}\left(y_{1}\right)-a^{2}\left(y_{2}\right)\right| & \leq \frac{e^{-2 L T}}{4 L|c| T^{2}}\left|y_{1}-y_{2}\right| .
\end{aligned}
$$

In addition, suppose that for $R=2 L T e^{L T}$ there exists a bounded solution to (5.4) with the terminal condition $\alpha(y, T)=0$. Then there exists a solution 
to (5.3) with the terminal condition $\alpha(y, T)=0$, which is bounded together with the first $y$-derivative.

Proof. Using standard verification theorems (see for instance Theorem 6.1 we obtain a stochastic representation of a solution to (5.4):

$$
\alpha(y, t)=\max _{\eta \in \mathcal{M}} \min _{q \in \mathcal{N}} \mathbb{E}\left[\int_{t}^{T}\left(h\left(Y_{s}^{\eta, q}(y, t), s\right)-c q_{s}^{2} a^{2}\left(Y_{s}^{\eta, q}(y, t)\right)\right) d s\right],
$$

where $\mathcal{N}$ is the set of all progressively measurable processes $q$ taking values in $[-R, R], \mathcal{M}$ is the set of all progressively measurable processes $\eta=\left(\eta_{1}, \eta_{2}\right)$ taking values in $\Gamma$, and the process $Y^{\eta, q}(y, t)$ is a unique strong solution to $(1)$

$$
\left\{\begin{array}{l}
d Y_{s}=\left[f\left(Y_{s}\right)+i\left(Y_{s}, \eta_{s}\right)+2 c a^{2}\left(Y_{s}\right) q_{s}\right] d t+a\left(Y_{s}\right) d W_{t}^{1} \\
Y_{t}=x
\end{array}\right.
$$

We have the following estimate:

$$
\begin{aligned}
& \left|\alpha\left(y_{1}, t\right)-\alpha\left(y_{2}, t\right)\right| \\
& \quad \leq T\left(L+\frac{e^{-2 L T}}{4 L|c| T^{2}}|c| R^{2}\right) \max _{\eta \in \Gamma} \max _{q \in[-R, R]} \mathbb{E} \sup _{t \leq s \leq T}\left|Y_{s}^{\eta, q}\left(y_{1}, t\right)-Y_{s}^{\eta, q}\left(y_{2}, t\right)\right| \\
& \quad \leq 2 L T \mathbb{E} \sup _{t \leq s \leq T}\left|Y_{s}^{\eta, q}\left(y_{1}, t\right)-Y_{s}^{\eta, q}\left(y_{2}, t\right)\right| .
\end{aligned}
$$

In addition, using Theorem 1.3.16 from Pham [17] we get

$$
\begin{aligned}
& \mathbb{E} \sup _{t \leq s \leq T}\left|Y_{s}^{\eta, q}\left(y_{1}, t\right)-Y_{s}^{\eta, q}\left(y_{2}, t\right)\right| \\
& \quad \leq 2 L T \exp \left(T \max \left(L, 2 R|c| \frac{e^{-2 L T}}{4 L|c| T^{2}}\right)\right)\left|y_{1}-y_{2}\right|=e^{L T}\left|y_{1}-y_{2}\right| .
\end{aligned}
$$

Therefore, $\left|\alpha_{y}\right| \leq 2 L T e^{L T}=R$ and so a solution to (5.4) is also a solution to

$$
\alpha_{t}+\frac{1}{2} a^{2}(y) \alpha_{y y}+c a^{2}(y) \alpha_{y}^{2}+f(y) \alpha_{y}+\max _{\eta \in \Gamma}\left(i(y, \eta) \alpha_{y}+h(y, \eta)\right)=0 .
$$

Remark. From the stochastic representation (5.5) uniqueness in the class of functions bounded together with the first $y$-derivative can also be obtained for solution to (5.6).

To ensure existence and uniqueness of a solution to (5.4) we use a classical result proved by Friedman [4].

TheOrem 5.2. Suppose that $H(t, y, u, p)$ is uniformly Lipschitz continuous in $(y, u, p)$ and uniformly Hölder continuous in $t$ in compact subsets of

$\left({ }^{1}\right)$ It is more convenient here to change our convention and use $Y^{\eta, q}(y, t)$ under $\mathbb{E}$ instead of a symbol $\mathbb{E}_{y, t}$. 
$[0, T] \times \mathbb{R} \times \mathbb{R} \times \mathbb{R}$, and let $H$ satisfy

$$
\begin{aligned}
& H(t, y, 0,0) \leq K, \\
& H(t, y, u, 0)-H(t, y, \bar{u}, 0) \leq K(u-\bar{u}) \quad \text { if } u>\bar{u}, \\
& H(t, y, u, p)-H(t, y, \bar{u}, p) \leq K_{R}(u-\bar{u}) \quad \text { if }|u|,|\bar{u}| \leq R, u>\bar{u}, \\
& |H(t, y, u, p)-H(t, \bar{y}, u, p)| \leq K_{R}(1+|p|)|y-\bar{y}| \quad \text { if }|u| \leq R, \\
& |H(t, y, u, p)-H(t, y, u, \bar{p})| \leq K_{R}(1+|y|)|p-\bar{p}| \quad \text { if }|u| \leq R,
\end{aligned}
$$

for any $R>0$ and $0 \leq t \leq T, y, \bar{y}, p, \bar{p} \in \mathbb{R}$. Moreover, let $\varphi$ be a bounded and uniformly Lipschitz continuous function. Then there exists a unique solution $u$ of the Cauchy problem

$$
\left\{\begin{array}{l}
u_{t}+\frac{1}{2} u_{y y}+H\left(t, y, u, u_{y}\right)=0 \\
u(y, T)=\varphi(y)
\end{array}\right.
$$

which is bounded together with the derivative $u_{y}$.

Collecting the above results together we easily get:

Proposition 5.3. If $a \equiv 1, i, f$ are continuous functions, $h$ is continuous and bounded and there exists $L>0$ such that

$$
\begin{aligned}
\left|f\left(y_{1}\right)-f\left(y_{2}\right)\right| & \leq L\left|y_{1}-y_{2}\right|, \\
\left|i\left(y_{1}, \eta\right)-i\left(y_{2}, \eta\right)\right| & \leq L\left|y_{1}-y_{2}\right|, \\
\left|h\left(y_{1}, \eta\right)-h\left(y_{2}, \eta\right)\right| & \leq L\left|y_{1}-y_{2}\right|,
\end{aligned}
$$

then there exists a solution to

$$
F_{t}+\frac{1}{2} a^{2}(y) F_{y y}+c a^{2}(y) \frac{F_{y}^{2}}{F}+f(y) F_{y}+\max _{\eta \in \Gamma}\left(i(y, \eta) F_{y}+h(y, \eta) F\right)=0
$$

with the terminal condition $F(y, T)=1$, which is positive, bounded together with $F_{y}$ and bounded away from zero.

Proof. Consider the function

where

$$
H(t, y, u, p):=\max _{\eta \in \Gamma} k_{1}(t, y, u, p, \eta)+\min _{q \in[-R, R]} k_{2}(t, y, u, p, q),
$$

$$
\begin{aligned}
& k_{1}(t, y, u, p, \eta):=i(y, \eta) p+h(y, \eta), \\
& k_{2}(t, y, u, p, q):=f(y)-(1 / 2+c) a^{2}(y) q^{2}+2(1 / 2+c) a^{2}(y) p q .
\end{aligned}
$$

The functions $k_{1}$ and $k_{2}$ do not depend on $u$ and are linear with respect to $p$. Therefore, from the assumed regularity of $i$ and $h$ and well known inequalities

$$
\begin{aligned}
\left|\max _{\eta} k(z, \eta)-\max _{\eta} k(\bar{z}, \eta)\right| & \leq \max _{\eta}|k(z, \eta)-k(\bar{z}, \eta)|, \\
\left|\min _{q} k(z, q)-\min _{q} k(\bar{z}, q)\right| & \leq \max _{q}|k(z, q)-k(\bar{z}, q)|,
\end{aligned}
$$

it follows that $H$ satisfies all conditions of Theorem 5.2 . 
Therefore there exists a classical solution to

$$
\alpha_{t}+\frac{1}{2} a^{2}(y) \alpha_{y y}+\left(\frac{1}{2}+c\right) a^{2}(y) \alpha_{y}^{2}+f(y) \alpha_{y}+\max _{\eta \in \Gamma}\left(i(y, \eta) \alpha_{y}+h(y, \eta)\right)=0
$$

with the terminal condition $\alpha(y, T)=0$, bounded together with the $y$-derivative.

Simple differentiation shows that $F(y, t):=e^{\alpha(y, t)}$ is a positive solution to 5.12 , bounded together with the $y$-derivative and bounded away from zero.

6. Appendix. Let us recall the objective function of our game:

$$
\mathcal{K}^{\pi, \eta}(x, y, t):=\mathbb{E}_{x, y, t}^{\eta}\left(e^{-\frac{1}{2} \theta \int_{t}^{T}\left|\eta_{s}\right|^{2} d s}\left(X_{T}^{\pi}-D\right)^{2}\right) .
$$

It is also useful to write here a general form of the differential operator which was used to solve our game problems:

$$
\begin{aligned}
\mathcal{H}^{\pi, \eta} V(x, y, t):= & V_{t}+\frac{1}{2} a^{2}(y) V_{y y}+\frac{1}{2} \pi^{2} \sigma^{2}(y) V_{x x}+\rho \pi \sigma(y) a(y) V_{x y} \\
& +\pi\left(b(y)-r+\eta_{1} \sigma(y)\right) V_{x}+\left(\rho \eta_{1}+\bar{\rho} \eta_{2}\right) a(y) V_{y}+g(y) V_{y} .
\end{aligned}
$$

If $\theta=0$, then $J^{\pi, \eta}(x, y, t)=\mathcal{K}^{\pi, \eta}(x, y, t)$ and $\mathcal{H}^{\pi, \eta}=\mathcal{L}^{\pi, \eta}$ and the proof of the Verification Theorem, given below, is valid also for Theorem 3.1. In fact, we do not give all details, since this is only a modification of standard results.

TheOREM 6.1. Suppose there exists a function $V \in \mathcal{C}^{2,2,1}\left(\mathbb{R}^{2} \times[0, T)\right) \cap$ $\mathcal{C}\left(\mathbb{R}^{2} \times[0, T]\right)$ and an admissible Markov control $\left(\pi^{*}(x, y, t), \eta^{*}(x, y, t)\right)$ such that

$$
\begin{aligned}
& \mathcal{H}^{\pi^{*}(x, y, t), \eta} V(x, y, t) \leq 0, \\
& \mathcal{H}^{\pi, \eta^{*}(x, y, t)} V(x, y, t) \geq 0 \\
& \mathcal{H}^{\pi^{*}(x, y, t), \eta^{*}(x, y, t)} V(x, y, t)=0, \\
& V(x, y, T)=(x-D)^{2} \quad \text { for all } \eta \in \Gamma, \pi \in \mathbb{R},(x, y, t) \in \mathbb{R}^{2} \times[0, T),
\end{aligned}
$$

and

$$
\begin{aligned}
& \mathbb{E}_{x, y, t}^{\eta}\left(\sup _{t \leq s \leq T} e^{-\frac{1}{2} \theta \int_{t}^{s}\left|\eta_{s}\right|^{2} d s}\left|V\left(X_{s}^{\pi}, Y_{s}, s\right)\right|\right)<\infty \\
& \qquad \text { for all }(x, y, t) \in \mathbb{R}^{2} \times[0, T], \pi \in \mathcal{A}_{t}, \eta \in \mathcal{M} .
\end{aligned}
$$

Then

$$
\mathcal{K}^{\pi^{*}, \eta}(x, y, t) \leq V(x, y, t) \leq \mathcal{K}^{\pi, \eta^{*}}(x, y, t) \quad \text { for all } \pi \in \mathcal{A}_{t}, \eta \in \mathcal{M},
$$

and

$$
V(x, y, t)=\mathcal{K}^{\pi^{*}, \eta^{*}}(x, y, t)
$$


Proof. Fix $(x, y, t) \in \mathbb{R}^{2} \times[0, T)$. Fix $\eta \in \mathcal{M}$ and consider the system

$$
\left\{\begin{array}{l}
d X_{t}=\pi^{*}\left(X_{t}, Y_{t}, t\right)\left(b\left(Y_{t}\right)-r\right) X_{t} d t+\pi^{*}\left(X_{t}, Y_{t}, t,\right) X_{t} \sigma\left(Y_{t}\right) d W_{t}^{1}, \\
d Y_{t}=g\left(Y_{t}\right) d t+a\left(Y_{t}\right)\left(\rho d W_{t}^{1}+\bar{\rho} d W_{t}^{2}\right) .
\end{array}\right.
$$

Using the Girsanov transformation we get the $Q^{\eta}$-dynamics of the system 6.6):

$$
\left\{\begin{array}{l}
d X_{t}=\pi_{t}^{*}\left(\left(b\left(Y_{t}\right)-r\right)+\sigma\left(Y_{t}\right) \eta_{1 t}\right) X_{t} d t+\pi_{t}^{*} \sigma\left(Y_{t}\right) X_{t} d W_{t}^{1 \eta} \\
d Y_{t}=\left(g\left(Y_{t}\right)+a\left(Y_{t}\right)\left(\rho \eta_{1 t}+\bar{\rho} \eta_{2 t}\right)\right) d t+a\left(Y_{t}\right)\left(\rho d W_{t}^{1 \eta}+\bar{\rho} d W_{t}^{2 \eta}\right),
\end{array}\right.
$$

where $\pi_{t}^{*}=\pi^{*}\left(X_{t}, Y_{t}, t\right)$, and $\left(W_{t}^{1 \eta}, W_{t}^{2 \eta}\right)^{T}$ is the $Q^{\eta}$-Brownian motion given by

$$
\left\{\begin{array}{l}
d W_{t}^{1 \eta}=d W_{t}^{1}-\eta_{1 t} d t \\
d W_{t}^{2 \eta}=d W_{t}^{2}-\eta_{2 t} d t .
\end{array}\right.
$$

If we apply the Itô formula to (6.7), the function $V$ and the process $e^{-\frac{1}{2} \theta \int_{t}^{s}\left|\eta_{k}\right|^{2} d k}$, we get

$$
\begin{aligned}
& \mathbb{E}_{x, y, t}^{\eta}\left(e^{-\frac{1}{2} \theta \int_{t}^{(T-\varepsilon) \wedge T_{n}^{\varepsilon}}\left|\eta_{k}\right|^{2} d k} V\left(X_{(T-\varepsilon) \wedge T_{n}^{\varepsilon}}, Y_{(T-\varepsilon) \wedge T_{n}^{\varepsilon}},(T-\varepsilon) \wedge T_{n}^{\varepsilon}\right)\right)=V(x, y, t) \\
& +\mathbb{E}_{x, y, t}^{\eta} \int_{t}^{(T-\varepsilon) \wedge T_{n}^{\varepsilon}} e^{-\frac{1}{2} \theta \int_{t}^{s}\left|\eta_{k}\right|^{2} d k} \mathcal{H}^{\pi_{s}^{*}, \eta_{s}} V\left(X_{s}, Y_{s}, s\right) d s+\mathbb{E}_{x, y, t}^{\eta} \int_{t}^{(T-\varepsilon) \wedge T_{n}^{\varepsilon}} M_{s}^{\varepsilon} d W_{s}^{\eta},
\end{aligned}
$$

where $\left(T_{n}^{\varepsilon}, n=1,2, \ldots\right)\left(T_{n}^{\varepsilon} \rightarrow \infty\right)$ is a localizing sequence of stopping times such that

$$
\mathbb{E}_{x, y, t}^{\eta} \int_{t}^{(T-\varepsilon) \wedge T_{n}^{\varepsilon}} M_{s}^{\varepsilon} d W_{s}^{\eta}=0 .
$$

Applying (6.1) yields

$\mathbb{E}_{x, y, t}^{\eta}\left(e^{-\frac{1}{2} \theta \int_{t}^{(T-\varepsilon) \wedge T_{n}^{\varepsilon}}\left|\eta_{k}\right|^{2} d k} V\left(X_{(T-\varepsilon) \wedge T_{n}^{\varepsilon}}, Y_{(T-\varepsilon) \wedge T_{n}^{\varepsilon}},(T-\varepsilon) \wedge T_{n}^{\varepsilon}\right)\right) \geq V(x, y, t)$.

Since (6.5) holds, we can apply the dominated convergence theorem. Letting $n \rightarrow \infty, \varepsilon \rightarrow 0$ and using (6.4) we obtain

$$
V(x, y, t) \geq \mathcal{K}^{\pi^{*}, \eta}(x, y, t) .
$$

If we replace $\eta$ by $\eta^{*}$ and apply (6.3), we get

$$
V(x, y, t)=\mathcal{K}^{\pi^{*}, \eta^{*}}(x, y, t) .
$$

Next we choose $\pi \in \mathcal{A}_{t}$ and apply the Itô formula to the system

$$
\left\{\begin{array}{l}
\left.d X_{t}=\pi_{t}\left(b\left(Y_{t}\right)-r\right)+\sigma\left(Y_{t}\right) \eta_{1 t}^{*}\right) X_{t} d t+\pi_{t} \sigma\left(Y_{t}\right) X_{t} d W_{t}^{1 \eta^{*}} d t, \\
d Y_{t}=\left(g\left(Y_{t}\right)+a\left(Y_{t}\right)\left(\rho \eta_{1 t}^{*}+\eta_{2 t}^{*} \bar{\rho}\right)\right) d t+a\left(Y_{t}\right)\left(\rho d W_{t}^{1 \eta^{*}}+\bar{\rho} d W_{t}^{2 \eta^{*}}\right) .
\end{array}\right.
$$

Repeating the procedure above and using $(6.2)$, we get

$$
V(x, y, t) \leq \mathcal{K}^{\pi, \eta^{*}}(x, y, t) .
$$


Acknowledgments. The author would like to thank the anonymous referee whose suggestions helped to improve first version of this paper.

\section{References}

[1] J. Cvitanić and I. Karatzas, On dynamic measures of risk, Finance Stoch. 3 (1999), $451-482$.

[2] Ł. Delong, Optimal investment strategy for a non-life insurance company: quadratic loss, Appl. Math. (Warsaw) 32 (2005), 263-277.

[3] K. Fan Minimax theorems, Proc. Nat. Acad. Sci. U.S.A. 39 (1953), 42-47.

[4] A. Friedman, The Cauchy problem for first order partial differential equations, Indiana Univ. Math. J. 23 (1973), 27-40.

[5] D. Goldfarb and G. Iyengar, Robust portfolio selection problems, Math. Oper. Res. 28 (2003), 1-38.

[6] A. Gundel, Robust utility maximization for complete and incomplete market models, Finance Stoch. 9 (2005), 151-176.

[7] D. Hernández and A. Schied, Robust utility maximization in a stochastic factor model, Statist. Decisions 24 (2006), 109-125.

[8] S. Jaimungal and G. Sigloch, Incorporating risk and ambiguity aversion into a hybrid model of default, Math. Finance 22 (2012), 57-81.

[9] D. Kahneman and A. Tversky, Prospect theory: an analysis of decision under risk, Econometrica 47 (1979), 263-291.

[10] J. Laurent and H. Pham, Dynamic programming and mean-variance hedging, Finance Stoch. 3 (1999), 83-110.

[11] P. Maenhout, Robust portfolio rules and detection-error probabilities for a meanreverting risk premium, J. Econom. Theory 128 (2006), 136-163.

[12] H. Markowitz, Portfolio selection, J. Finance 7 (1952), 77-91.

[13] S. Mataramvura and B. Øksendal, Risk minimizing portfolios and HJBI equations for stochastic differential games, Stochastics 80 (2008), 317-337.

[14] B. Øksendal and A. Sulem, A game theoretic approach to martingale measures in incomplete markets, Surveys Appl. Industrial Math. 15 (2008), 18-24.

[15] B. Øksendal and A. Sulem, Risk indifference pricing in jump diffusion markets, Math. Finance 19 (2009), 619-637.

[16] H. Pham, Smooth solutions to optimal investment models with stochastic volatilities and portfolio constraints, Appl. Math. Optim. 46 (2002), 55-78.

[17] H. Pham, Continuous-Time Stochastic Control and Optimization with Financial Applications, Stochastic Modelling Appl. Probab. 61, Springer, Berlin, 2009.

[18] A. Schied, Robust optimal control for a consumption-investment problem, Math. Methods Oper. Res. 67 (2008), 1-20.

[19] A. Schied and C. T. Wu, Duality theory for optimal investments under model uncertainty, Statist. Decisions 23 (2005), 199-217.

[20] D. Talay and Z. Zheng, Worst case model risk management, Finance Stoch. 6 (2002), $517-537$.

[21] R. H. Tütüncu and M. Koenig, Robust asset allocation, Ann. Oper. Res. 132 (2004), $157-187$.

[22] S. Xie, Continuous-time mean-variance portfolio selection with liability and regime switching, Insurance Math. Econom. 45 (2009), 148-155.

[23] T. Zariphopoulou, A solution approach to valuation with unhedgeable risks, Finance Stoch. 5 (2001), 61-82. 
[24] D. Zawisza, Robust portfolio selection under exponential preferences, Appl. Math. (Warsaw) 37 (2010), 215-230.

[25] X. Y. Zhou and D. Li, Continuous-time mean-variance portfolio selection: a stochastic LQ framework, Appl. Math. Optim. 42 (2000), 19-33.

[26] W. Zhu, Ambiguity aversion and an intertemporal equilibrium model of catastrophelinked securities pricing, Insurance Math. Econom. 49 (2011), 38-46.

Dariusz Zawisza

Institute of Mathematics

Faculty of Mathematics and Computer Science

Jagiellonian University

Łojasiewicza 6

30-348 Kraków, Poland

E-mail: dariusz.zawisza@im.uj.edu.pl

Received on 4.1.2012;

revised version on 10.5.2012 
